\title{
Bargaining with non-convexities
}

Citation for published version (APA):

Herings, P. J. J., \& Predtetchinski, A. (2009). Bargaining with non-convexities. METEOR, Maastricht University School of Business and Economics. METEOR Research Memorandum No. 042 https://doi.org/10.26481/umamet.2009042

Document status and date:

Published: 01/01/2009

DOI:

10.26481/umamet.2009042

Document Version:

Publisher's PDF, also known as Version of record

\section{Please check the document version of this publication:}

- A submitted manuscript is the version of the article upon submission and before peer-review. There can be important differences between the submitted version and the official published version of record.

People interested in the research are advised to contact the author for the final version of the publication, or visit the DOI to the publisher's website.

- The final author version and the galley proof are versions of the publication after peer review.

- The final published version features the final layout of the paper including the volume, issue and page numbers.

Link to publication

\footnotetext{
General rights rights.

- You may freely distribute the URL identifying the publication in the public portal. please follow below link for the End User Agreement:

www.umlib.nl/taverne-license

Take down policy

If you believe that this document breaches copyright please contact us at:

repository@maastrichtuniversity.nl

providing details and we will investigate your claim.
}

Copyright and moral rights for the publications made accessible in the public portal are retained by the authors and/or other copyright owners and it is a condition of accessing publications that users recognise and abide by the legal requirements associated with these

- Users may download and print one copy of any publication from the public portal for the purpose of private study or research.

- You may not further distribute the material or use it for any profit-making activity or commercial gain

If the publication is distributed under the terms of Article $25 \mathrm{fa}$ of the Dutch Copyright Act, indicated by the "Taverne" license above, 


\section{Maastricht University}

P. Jean-J acques Herings,

Arkadi Predtetchinski

Bargaining with Non-convexities

RM/09/042

\section{METEOR}

Maastricht University School of Business and Economics

Maastricht Research School of Economics

of Technology and Organization

PO. Box 616

NL-6200 MD Maastricht

The Netherlands 


\title{
Bargaining with Non-convexities
}

\author{
P. Jean-Jacques Herings* $\quad$ Arkadi Predtetchinski ${ }^{\dagger}$
}

October 8, 2009

\begin{abstract}
We show that in the canonical non-cooperative multilateral bargaining game, a subgame perfect equilibrium exists in pure stationary strategies, even when the space of feasible payoffs is not convex. At such an equilibrium there is no delay. We also have the converse result that randomization will not be used in this environment in the sense that all stationary subgame perfect equilibria do not involve randomization on the equilibrium path. Nevertheless, mixed strategy profiles can lead to Pareto superior payoffs in non-convex cases.
\end{abstract}

KEYWORDS: Bargaining, non-convexities, stationary subgame perfect equilibrium, existence, pure strategies.

JEL CODES: C62, C72, C73, C78.

*P.J.J. Herings, Department of Economics, Maastricht University, P.O. Box 616, 6200 MD, Maastricht, The Netherlands. This author would like to thank the Netherlands Organization for Scientific Research (NWO) for financial support. P.Herings@maastrichtuniversity.nl

${ }^{\dagger}$ A. Predtetchinski, Department of Economics, Maastricht University, P.O. Box 616, 6200 MD, Maastricht, The Netherlands. This author would like to thank the Netherlands Organization for Scientific Research (NWO) for financial support. A.Predtetchinski@maastrichtuniversity.nl 


\section{Introduction}

Many problems in economics are complicated by the presence of non-convexities. Scarf (1994) mentions the omnipresence of non-divisibilities in production as an important source of non-convexities in economics. Another example of a non-convexities in production is the existence of production technologies with increasing returns to scale. Other important cases of non-convexities result from non-convexities in preferences, even in the presence of lotteries when agents are not expect utility maximizers as is for instance the case in prospect theory, see Kahneman and Tversky (1979), or when randomization is not possible, and non-convexities in the consumption set, for instance caused by the presence of indivisible commodities. Although non-convexities are regarded important, most of the economic literature assumes them away for reasons of intractability.

Non-convexities are frequently studied in the $n$-person cooperative bargaining literature. There is for instance an extensive literature on the extension of the Nash bargaining solution to non-convex environments (Kaneko (1980), Conley and Wilkie (1996), Mariotti (1997), Zhou (1997), and Xu and Yoshihara (2006)). On the contrary, the literature on strategic bargaining has not paid much attention to non-convexities and non-convexities are only treated for the two-player case. Rubinstein (1982) allows for modest forms of non-convexities. Under his hypothesis there is typically a unique equilibrium. Herrero (1989) considers general non-convexities for the two-player case and obtains the existence of a subgame perfect equilibrium in stationary strategies when the set of feasible payoffs is strictly comprehensive. Conley and Wilkie (1995) also consider a strictly comprehensive set of feasible payoffs and introduce a bargaining protocol that implements their extension of the Nash bargaining solution.

Existence of a pure equilibrium in the canonical multilateral bargaining model has been shown only when the set of feasible payoffs is convex. The existence of such an equilibrium has been shown in Banks and Duggan (2000). Merlo and Wilson (1995) consider the $n$-person cake division problem and obtain the existence of a unique subgame perfect equilibrium in stationary strategies when the set of feasible payoffs is convex and the proposer selection protocol is deterministic.

We consider the following canonical multilateral bargaining procedure. In each time period, nature randomly selects a player that is allowed to make a proposal. All players respond sequentially to the proposal and either vote in favor or against. As soon as a responder votes against the proposal, the procedure continues in the next period. If all responders vote in favor of the proposal, it is accepted, and the procedure ends.

The bargaining game is fully characterized by the set of players, their discount factors, the set of feasible payoffs, and the probability according to which nature selects a particular proposer. The only assumptions we make regarding the set of feasible alternatives are non- 
substantial technical ones. It is assumed to be closed, comprehensive from below, and the set of individually rational payoffs is assumed bounded from above. To make the bargaining problem non-trivial, it is assumed that there is an alternative that gives all players a strictly positive payoff.

We show that this entire class of bargaining games has stationary subgame perfect equilibria in pure strategies that ensure immediate agreement. This result is surprising as the usual way to deal with non-convexities is to introduce lotteries. For that reason, one might have expected that non-convex bargaining games possess mixed strategy equilibria rather than pure ones. Similarly, one might have expected that non-convexities are a potential source for delay.

We also address the reverse question. Under what conditions are all stationary subgame perfect equilibria of a bargaining game in pure strategies without delay? The answer is that an extremely mild additional assumption assures this: When the set of weakly Pareto optimal alternatives coincides with the set of Pareto optimal ones, all stationary subgame perfect equilibria involve no randomization on the equilibrium path. Equilibria are characterized by the absence of delay.

To derive the first main result, the existence result, we deviate from the usual proof strategy that basically exploits continuity of the best-response correspondences. In our non-convex setting, this correspondence may not be continuous. Instead, we construct an excess utility function that resembles the excess demand function as used in general equilibrium theory. Let some profile of ex ante utilities be given and consider for each player $i$ the (potentially infeasible) proposal player $i$ has to make in order to be consistent with this profile of ex ante utilities. Coordinate $i$ of the excess utility function is the degree of feasibility of this proposal. The excess utility function is shown to have a zero point by showing that it is not outward pointing. Next, a zero point is shown to induce a subgame perfect equilibrium in stationary strategies of the bargaining game.

To prove the second main result, roughly stating that all stationary subgame perfect equilibria are in pure strategies, we proceed in several steps. One of the main steps is to show in an equilibrium in mixed strategies, proposals offering strictly more than the continuation utility to all players are accepted with probability one, whereas proposals offering at least one player strictly less than the continuation utility are accepted with probability zero. The next main step is to argue that for every player there is a unique proposal which maximizes his utility subject to being accepted with probability one and that every mixed equilibrium puts probability one on such a proposal.

Equilibria are efficient in the sense that in equilibrium every proposer selects a Pareto optimal alternative. However, the fact that all equilibria are in pure strategies implies that equilibria may be inefficient in a weaker sense. It is not difficult to construct examples and 
mixed strategy profiles with associated utilities strictly Pareto dominating the equilibrium utilities.

This paper is organized as follows. Section 2 introduces the model and Section 3 provides the existence result. Section 4 proves the converse result and Section 5 concludes.

\section{The Bargaining Game}

We consider the bargaining game $\Gamma=(N, V, \delta, \theta)$. There is a set $N$ of $n$ individuals that have to select a single payoff vector in the set of feasible payoffs $V$, a non-empty subset of $\mathbb{R}^{N}$. We denote the set of non-negative feasible payoffs by $V_{+}=V \cap \mathbb{R}_{+}^{N}$. The vector $\delta=\left(\delta_{i}\right)_{i \in N}$ consists of the players' discount factors and $\theta=\left(\theta_{i}\right)_{i \in N}$ denotes the probability according to which players are selected as a proposer. Individuals negotiate about the alternative to be selected using a procedure defined as follows.

In every time period $t$, starting with $t=0$, nature selects player $i$ to be the proposer with probability $\theta_{i}$. After history $h$, player $i$ makes a proposal $\sigma^{i}(h)$. In Sections 2 and 3 we restrict ourselves to pure strategies, in which case $\sigma^{i}(h)$ corresponds to an element in $V_{+}$. After observing $\sigma^{i}(h)$, all players (including the proposer) vote sequentially on the proposal, the order of their responses being history independent. Each player can either accept or reject the proposal. If the proposal is unanimously accepted, the proposal $\sigma^{i}(h)$ is implemented. As soon as the first rejection occurs, the period $t+1$ begins, with nature selecting a new proposer, and so on.

The utility to player $i$ of agreement on $v \in V$ in time period $t$ is given by $\delta_{i}^{t} v_{i}$. The utility of perpetual disagreement is equal to 0 for all players. Players have von NeumannMorgenstern utility functions.

Our assumptions are as follows.

(A) The set $V$ is closed and comprehensive from below. The set $V_{+}$is bounded and contains 0 in its interior.

(B) The discount factor $\delta_{i}$ belongs to $[0,1)$ for all $i$.

We analyze the stationary subgame perfect equilibria (SSPE) of $\Gamma$. A stationary strategy of individual $i$ specifies a proposal $x^{i}$ and an individual acceptance set $A^{i}$. At every history $h$ where player $i$ is selected as a proposer, he makes the proposal $\sigma^{i}(h)=x^{i}$ and at every history $h$ where player $i$ has to respond, he accepts the proposal currently on the table if and only if it belongs to $A^{i}$. A strategy profile is a stationary subgame perfect equilibrium if it is stationary and if it induces a Nash equilibrium in every subgame.

The main existence result for this model is Theorem 2 in Banks and Duggan (2000). This theorem states that when the set of feasible payoffs is convex, then there exists a pure 
strategy no-delay stationary equilibrium. Moreover, they have the converse result that every no-delay stationary equilibrium is in pure strategies when the set of feasible payoffs is strictly convex. We show that for an existence result, it suffices to make assumptions (A) and (B). It follows in particular that convexity assumptions are not needed. Under the modest additional assumption that weakly Pareto efficient and Pareto efficient points coincide, we obtain the converse result that every stationary equilibrium uses pure strategies on the equilibrium path and does not involve delay.

\section{Existence of Stationary Subgame Perfect Equilibria in Pure Strategies}

In this section we present a system of equations which is such that a solution to the system induces an SSPE in pure strategies. Let $g: \mathbb{R}^{N} \rightarrow \mathbb{R}$ be a transformation function, i.e. a function such that $v \in V$ if and only if $g(v) \geq 0$, with $v \in \partial V$ if and only if $g(v)=0$.

Assumption A1 can be rephrased in terms of $g$. It is evident that A1 is equivalent to the existence of a transformation function $g$ satisfying the following conditions:

- $g$ is continuous,

- $g\left(0^{N}\right)>0$,

- $g(\hat{v}) \geq 0$ and $v \leq \hat{v}$ implies $g(v) \geq 0$,

- $g^{-1}\left(\mathbb{R}_{+}\right) \cap \mathbb{R}_{+}^{N}$ is bounded.

Let $u \in \mathbb{R}^{N}$ be a vector of ex ante expected utilities for the players. Consider the case where for every player $i$ his proposal $x^{i}$ is immediately accepted and satisfies $g\left(x^{i}\right)=0$ and $x_{j}^{i}=\delta_{j} u_{j}$ for $j \neq i$. Player $i$ is the proposer with probability $\theta_{i}$ and is a responder with probability $1-\theta_{i}$, so consistency with ex ante expected utility imposes

$$
u_{i}=\theta_{i} x_{i}^{i}+\left(1-\theta_{i}\right) \delta_{i} u_{i}
$$

By rearranging terms we obtain that

$$
x_{i}^{i}=\frac{1-\delta_{i}+\theta_{i} \delta_{i}}{\theta_{i}} u_{i} .
$$

For $i \in N$, we define $\alpha_{i}=\left(1-\delta_{i}+\theta_{i} \delta_{i}\right) / \theta_{i}$ and we define the function $p^{i}: \mathbb{R}^{N} \rightarrow \mathbb{R}^{N}$ by

$$
p_{j}^{i}(u)= \begin{cases}\alpha_{i} u_{i}, & \text { if } j=i, \\ \delta_{j} u_{j}, & \text { if } j \in N \backslash\{i\}\end{cases}
$$


The function $p^{i}$ specifies the proposals that are consistent with ex ante expected utilities $u$.

Let $\bar{v} \in \mathbb{R}_{++}^{N}$ be a strict upper bound on $V_{+}$, so every $v \in V_{+}$satisfies $v \leq \bar{v}$.

We define the function $z:\left[0^{N}, \bar{v}\right] \rightarrow \mathbb{R}^{N}$ as follows:

$$
z_{i}(u)=g\left(p^{i}(u)\right), \quad i \in N .
$$

If $z_{i}(u)<0$, then there is no payoff vector in the set of feasible payoffs that gives player $i$ a payoff of $\alpha_{i} u_{i}$ and players $j \neq i$ a payoff of $\delta_{j} u_{j}$. Consistency with ex ante utilities would impose that $u_{i}$ be lowered. If $z_{i}(u)>0$, then there is a payoff vector in $V$ that gives player $i$ a payoff of strictly more than $\alpha_{i} u_{i}$ and players $j \neq i$ a payoff of $\delta_{j} u_{j}$. In this case it is possible to increase $u_{i}$.

The function $z$ is related to an excess demand function as used in general equilibrium theory and we can think of $z$ as an excess utility function. To find equilibria in pure strategies, we are looking for solutions to the system of equations $z(u)=0^{N}$. Notice that the system of equations $z(u)=0^{N}$ is different from the usual one (see Merlo and Wilson (1995), Banks and Duggan (2000), Kalandrakis (2004, 2006)), where typically each player is maximizing his utility subject to meeting the reservation values. Our assumptions do not imply that the system of equations employed in the usual approach is continuous.

A function is outward pointing at a point if the function value is a non-zero element of the normal cone at the point. A function is outward pointing if it is outward pointing at some point. When applied to a function $f:[\underline{a}, \bar{a}] \rightarrow \mathbb{R}^{m}$, where $[\underline{a}, \bar{a}]$ is the $m$-dimensional unit interval with $\underline{a} \ll \bar{a}$, we obtain the following definition.

Definition 3.1 Let $\underline{a}, \bar{a}$ in $\mathbb{R}^{m}$ be such that $\underline{a} \ll \bar{a}$. The function $f:[\underline{a}, \bar{a}] \rightarrow \mathbb{R}^{m}$ is outward pointing at $a \in[\underline{a}, \bar{a}]$ if $f(a) \neq 0$ and, for $k=1, \ldots, m, a_{k}=\underline{a}_{k}$ implies $f_{k}(a) \leq 0$, $\underline{a}_{k}<a_{k}<\bar{a}_{k}$ implies $f_{k}(a)=0$, and $a_{k}=\bar{a}_{k}$ implies $f_{k}(a) \geq 0$.

We show next that the excess utility function $z$ is not outward pointing.

LEMmA 3.2 The excess utility function $z$ is not outward pointing.

Proof: Clearly, $z$ is not outward pointing at any $u$ in the interior of $\left[0^{N}, \bar{v}\right]$.

Consider $u$ in the boundary of $\left[0^{N}, \bar{v}\right]$ and $i \in N$ such that $u_{i}=\bar{v}_{i}$. Then, since $p^{i}(u) \in \mathbb{R}_{+}^{N}$ and there is no $v \in V_{+}$with $v_{i}=\alpha_{i} \bar{v}_{i}>\bar{v}_{i}$, we have that

$$
z_{i}(u)=g\left(p^{i}(u)\right)<0 .
$$

Therefore $z$ is not outward pointing at such a point $u$.

Consider $u$ in the boundary of $\left[0^{N}, \bar{v}\right]$ such that $u \ll \bar{v}$ and $u_{i}=0$ for at least one $i \in N$. Suppose that $z$ is outward pointing at $u$. Clearly $u \neq 0^{N}$, since $z\left(0^{N}\right)=$ 
$\left(g\left(0^{N}\right), \ldots, g\left(0^{N}\right)\right) \gg 0^{N}$. Let $j$ be such that $0<u_{j}<\bar{v}_{j}$. Then, since $z$ is outward pointing at $u$,

$$
0=z_{j}(u)=g\left(p^{j}(u)\right)
$$

so $p^{j}(u) \in V$. Since $p^{j}(u)>p^{i}(u)$ for any $i \in N$ such that $u_{i}=0$, we have $p^{i}(u) \in V$, so $z_{i}(u)=g\left(p^{i}(u)\right) \geq 0$. Since $z$ is outward pointing at $u$ it follows that $z_{i}(u)=0$ for every $i$ such that $u_{i}=0$. We find that $z(u)=0$, a contradiction to $z$ being outward pointing at $u$.

Q.E.D.

Functions that are not outward pointing have a zero point.

Lemma 3.3 Let $\underline{a}, \bar{a}$ in $\mathbb{R}^{m}$ be such that $\underline{a} \ll \bar{a}$ and let the function $f^{0}:[\underline{a}, \bar{a}] \rightarrow \mathbb{R}^{m}$ be not outward pointing. Then $f^{0}$ has a zero point.

Proof: We define the function $f^{1}:\left[\underline{a}-1^{m}, \bar{a}+1^{m}\right] \rightarrow \mathbb{R}^{m}$ by

$$
f^{1}(a)=\lambda(a)\left(\pi_{[\underline{a}, \bar{a}]}(a)-a\right)+(1-\lambda(a)) f^{0}\left(\pi_{[\underline{a}, \bar{a}]}(a)\right), \quad a \in\left[\underline{a}-1^{m}, \bar{a}+1^{m}\right],
$$

where $\pi_{[\underline{a}, \bar{a}]}$ is the orthogonal projection function on $[\underline{a}, \bar{a}]$ and

$$
\lambda(a)=\left\|\pi_{[\underline{a}, \bar{a}]}(a)-a\right\|_{\infty}, \quad a \in\left[\underline{a}-1^{m}, \bar{a}+1^{m}\right],
$$

so the function $\lambda:\left[\underline{a}-1^{m}, \bar{a}+1^{m}\right] \rightarrow[0,1]$ measures the distance in infinity norm from the point $a$ to its projection. The function $\lambda$ is continuous and has the property that it is equal to 1 on the boundary of $\left[\underline{a}-1^{m}, \bar{a}+1^{m}\right]$, equal to 0 on $[\underline{a}, \bar{a}]$, and strictly in between 0 and 1 everywhere else. We define the function $f^{2}:\left[\underline{a}-1^{m}, \bar{a}+1^{m}\right] \rightarrow\left[\underline{a}-1^{m}, \bar{a}+1^{m}\right]$ by

$$
f^{2}(a)=\pi_{\left[\underline{a}-1^{m}, \bar{a}+1^{m}\right]}\left(a+f^{1}(a)\right), \quad a \in\left[\underline{a}-1^{m}, \bar{a}+1^{m}\right] .
$$

The function $f^{2}$ has a fixed point, say $a^{*}$, by Brouwer's fixed point theorem.

Suppose $a^{*}$ belongs to the boundary of $\left[\underline{a}-1^{m}, \bar{a}+1^{m}\right]$, i.e. $\lambda\left(a^{*}\right)=1$. Then

$$
a^{*}=f^{2}\left(a^{*}\right)=\pi_{\left[\underline{a}-1^{m}, \bar{a}+1^{m}\right]}\left(a^{*}+f^{1}\left(a^{*}\right)\right)=\pi_{\left[\underline{a}-1^{m}, \bar{a}+1^{m]}\right]}\left(a^{*}+\pi_{[\underline{a}, \bar{a}]}\left(a^{*}\right)-a^{*}\right)=\pi_{[\underline{a}, \bar{a}]}\left(a^{*}\right) \neq a^{*},
$$

a contradiction. It follows that $a^{*}$ is not in the boundary of the set $\left[\underline{a}-1^{m}, \bar{a}+1^{m}\right]$, i.e. it belongs to its relative interior. From this it follows that

$$
a^{*}=\pi_{\left[\underline{a}-1^{m}, \bar{a}+1^{m}\right]}\left(a^{*}+f^{1}\left(a^{*}\right)\right)=a^{*}+f^{1}\left(a^{*}\right),
$$

so $f^{1}\left(a^{*}\right)=0^{m}$. Using the definition of $f^{1}$ it then follows that

$$
f^{0}\left(\pi_{[\underline{a}, \bar{a}]}\left(a^{*}\right)\right)=-\frac{\lambda\left(a^{*}\right)}{1-\lambda\left(a^{*}\right)}\left(\pi_{[\underline{a}, \bar{a}]}\left(a^{*}\right)-a^{*}\right) .
$$


Now if $a^{*}$ is not an element of the set $[\underline{a}, \bar{a}]$, then $0<\lambda\left(a^{*}\right)<1$ and the vector $a^{*}-\pi_{[\underline{a}, \bar{a}]}\left(a^{*}\right)$ is a non-zero element of the normal cone of $[\underline{a}, \bar{a}]$ at the point $\pi_{[\underline{a}, \bar{a}]}\left(a^{*}\right)$. But this means that $f^{0}$ is outward pointing at $\pi_{[\underline{a}, \bar{a}]}\left(a^{*}\right)$, a contradiction. We conclude that $a^{*} \in[\underline{a}, \bar{a}]$. Thus $\lambda\left(a^{*}\right)=0$ and $\pi_{[\underline{a}, \bar{a}]}\left(a^{*}\right)=a^{*}$, so $a^{*}$ is a zero point of the function $f^{0}$.

Q.E.D.

\section{Corollary 3.4 The excess utility function z has a zero point.}

A zero point $u^{*}$ of $z$ induces a subgame perfect equilibrium in stationary strategies of $\Gamma$ as follows. For $i \in N$, we define

$$
x^{* i}=p^{i}\left(u^{*}\right),
$$

and

$$
\left.A^{* i}=\left\{v \in V \mid v_{i}>\delta_{i} u_{i}^{*}\right\} \cup\left\{v \in V \mid v_{i}=\delta_{i} u_{i}^{*} \text { and } \forall j \neq i, v_{j} \leq \alpha_{j} u_{j}^{*}\right)\right\}
$$

Whenever a player $i$ has to propose, he makes the proposal $x^{* i}$. Whenever a player $i$ has to respond, he accepts proposals that offer him strictly more utility than $\delta_{i} u_{i}^{*}$ or that offer him exactly $\delta_{i} u_{i}^{*}$ and do not offer more than $\alpha_{j} u_{j}^{*}$ for other players $j$. In this construction we exploit the degree of freedom that we have in case a player is indifferent between accepting and rejecting a proposal. This freedom is needed in particular when the set $V$ contains points that are only weakly Pareto optimal as Example 3.6 illustrates. Indeed, it may well be that a proposal that maximizes the proposer's utility subject to offering the other players their reservation values is not compatible with an equilibrium. Equilibrium play may require a proposer to settle for a weakly Pareto optimal proposal that is not Pareto optimal.

THEOREM 3.5 The strategy profile $\left(x^{*}, A^{*}\right)$ is an SSPE of $\Gamma$.

PROOF: It is well-known that the game $\Gamma$ has the one-shot deviation property, meaning that if there is a subgame where a player has some profitable deviation from a stationary strategy profile, then there must also be a subgame where this player has a profitable oneshot deviation, i.e. a single deviation by this player at the root of the subgame. A minor extension of the arguments in Fudenberg and Tirole (1991) would prove this point.

We verify that no player has a profitable one-shot deviation. Suppose at some history $h$ at time period $t$, player $i$ is proposer and makes proposal $x^{i}$, potentially different from $x^{* i}$. Notice that $x^{* i}$ is accepted leading to expected utility $\delta_{i}^{t} \alpha_{i} u_{i}^{*}$ for player $i$. If $x^{i} \notin \cap_{i \in N} A^{* i}$, then it will be rejected, leading to an expected utility of $\delta_{i}^{t+1} u_{i}^{*}<\delta_{i}^{t} \alpha_{i} u_{i}^{*}$. If $x^{i} \in \cap_{i \in N} A^{* i}$ it will be accepted, leading to a utility of $\delta_{i}^{t} x_{i}^{i}$. Since $x^{i} \in A^{* j}$ for $j \neq i$, it follows that $x_{i}^{i} \leq \alpha_{i} u_{i}^{*}$, so the expected utility to player $i$ of proposing $x^{i}$ is less than or equal to the expected utility of proposing $x^{* i}$. 
Suppose at some history $h$ at time period $t$, player $i$ is responder to a proposal $v \in A^{* i}$. In equilibrium, player $i$ accepts. The expect utility to player $i$ is $\delta_{i}^{t} v_{i}$ if the other players accept and $\delta_{i}^{t+1} u_{i}^{*}$ if some other player rejects, so expected utility weakly exceeds $\delta_{i}^{t+1} u_{i}^{*}$ in any case. Would player $i$ deviate to a rejection, then his expected utility equals $\delta_{i}^{t+1} u_{i}^{*}$. Consider a proposal $v \notin A_{i}^{*}$, so in particular $v_{i} \leq \delta_{i} u_{i}^{*}$. In equilibrium, player $i$ rejects, leading to expected utility $\delta_{i}^{t+1} u_{i}^{*}$. A deviation to acceptance leads to expected utility $\delta_{i}^{t} v_{i}$ if others accept and $\delta_{i}^{t+1} u_{i}^{*}$ if some other player rejects. In either case, expected utility is bounded above by $\delta_{i}^{t+1} u_{i}^{*}$.

Q.E.D.

EXAMPLE 3.6 Consider the set of feasible payoffs

$$
V=\left\{v \in \mathbb{R}^{2} \mid v_{1} \leq 2, v_{2} \leq 2, \max \left\{v_{1}, v_{2}\right\} \leq 1\right\},
$$

which is depicted in Figure 1. An upper bound on $V_{+}$is given by $\bar{v}=(2,2)$.

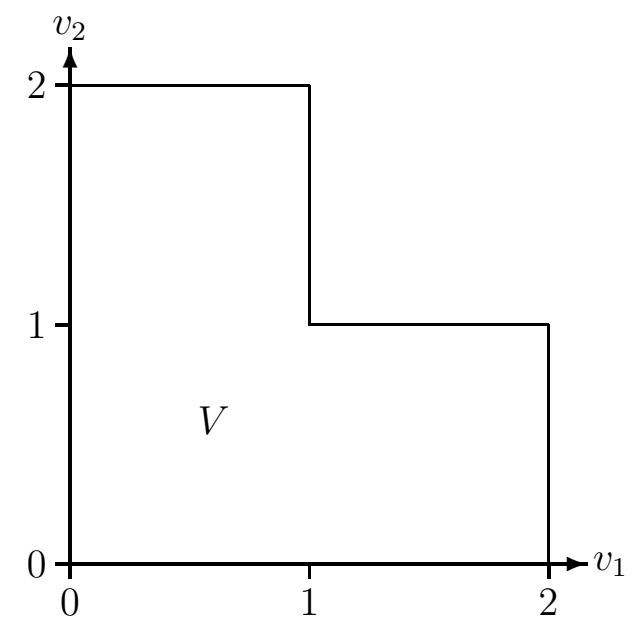

Figure 1: The set of feasible payoffs

Players are selected as proposer with equal probability, so $\theta_{1}=\theta_{2}=1 / 2$. It follows that $\alpha_{i}=2-\delta_{i}$. The excess utility function $z$ is then defined by

$$
\begin{array}{ll}
z_{1}(u)=g\left(p^{1}(u)\right)=g\left(\left(2-\delta_{1}\right) u_{1}, \delta_{2} u_{2}\right), & u \in[0, \bar{v}] \\
z_{2}(u)=g\left(p^{2}(u)\right)=g\left(\delta_{1} u_{1},\left(2-\delta_{2}\right) u_{2}\right), & u \in[0, \bar{v}] .
\end{array}
$$

The boundary of $V$ consists of four types of line segments, characterized by $v_{1} \leq 1$ and $v_{2}=2, v_{1}=1$ and $1 \leq v_{2} \leq 2,1 \leq v_{1} \leq 2$ and $v_{2}=1$, and $v_{1}=2$ and $0 \leq v_{2} \leq 1$. Since in equilibrium both $p^{1}(u)$ and $p^{2}(u)$ belong to such a line segment, there are potentially sixteen types of equilibria, where a type of equilibrium corresponds to a particular combination of line segments to which the proposal belongs. When we solve for $z(u)=0$ for each of the sixteen resulting systems of equations, and taking into account that $0 \leq \delta_{1}, \delta_{2}<1$, we find ex ante equilibrium utility levels $u_{1}^{*}$ and $u_{2}^{*}$. From these, we can derive the equilibrium 
proposals $x^{* 1}$ and $x^{* 2}$ and the equilibrium acceptance sets $A^{* 1}$ and $A^{* 2}$. The equilibrium proposals are displayed in Table 3 . We distinguish six types of equilibrium, labeled by A to $\mathrm{F}$. The conditions on the discount factors that lead to a particular equilibrium type are displayed in Table 3 and depicted in Figure 3.

\begin{tabular}{|l|l|l|l|l|}
\hline & Discount factors & $x^{* 1}$ & $x^{* 2}$ & \\
\hline $\mathrm{A}$ & $0 \leq \delta_{1}<1, \frac{2}{3}<\delta_{2}<1$ & $\left(1, \frac{2 \delta_{2}}{2-\delta_{2}}\right)$ & $\left(\frac{\delta_{1}}{2-\delta_{1}}, 2\right)$ & \\
$\mathrm{B}$ & $0 \leq \delta_{1}<1, \delta_{2}=\frac{2}{3}$ & $\left(\left(2-\delta_{1}\right) u_{1}^{*}, 1\right)$ & $\left(\delta_{1} u_{1}^{*}, 2\right)$ & $\frac{1}{2-\delta_{1}} \leq u_{1}^{*} \leq \min \left\{\frac{2}{2-\delta_{1}}, \frac{1}{\delta_{1}}\right\}$ \\
$\mathrm{C}$ & $0 \leq \delta_{1}<\frac{2}{3}, 0 \leq \delta_{2}<\frac{2}{3}$ & $\left(2, \frac{2 \delta_{2}}{2-\delta_{2}}\right)$ & $\left(\frac{2 \delta_{1}}{2-\delta_{1}}, 2\right)$ & \\
$\mathrm{D}$ & $\delta_{1}>\frac{2}{3}, \delta_{2}>\frac{2}{3}$ & $\left(\frac{2-\delta_{1}}{\delta_{1}}, 1\right)$ & $\left(1, \frac{2-\delta_{2}}{\delta_{2}}\right)$ & \\
$\mathrm{E}$ & $\delta_{1}=\frac{2}{3}, 0 \leq \delta_{2}<1$ & $\left(2, \delta_{2} u_{2}^{*}\right)$ & $\left(1,\left(2-\delta_{2}\right) u_{2}^{*}\right)$ & $\frac{1}{2-\delta_{2}} \leq u_{2}^{*} \leq \min \left\{\frac{2}{2-\delta_{2}}, \frac{1}{\delta_{2}}\right\}$ \\
$\mathrm{F}$ & $\frac{2}{3}<\delta_{1}<1,0 \leq \delta_{2}<1$ & $\left(2, \frac{\delta_{2}}{2-\delta_{2}}\right)$ & $\left(\frac{2 \delta_{1}}{2-\delta_{1}}, 1\right)$ & \\
\hline
\end{tabular}

Table 1: A summary of the possible equilibrium proposals.

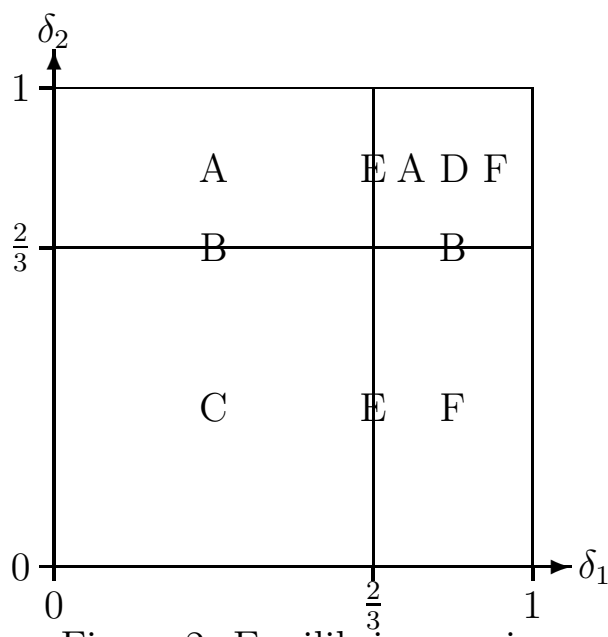

Figure 2: Equilibrium regions

Table 1 in conjunction with Figure 3 shows that equilibria are unique for discount factors in Regions A, C, and F in case at least one player has a discount factor below $2 / 3$ and no player has a discount factor equal to $2 / 3$. When the discount factor of at least one player is exactly equal to $2 / 3$, we are in Regions $\mathrm{B}$ or $\mathrm{E}$, and there are infinitely many equilibria and infinitely many possible equilibrium utilities. Finally, when both players have a discount factor above 2/3, equilibria of type A, D, and F co-exist. Figure 3 illustrates a typical combination of proposals for the various types of equilibria. Figure 4 illustrates the three possible equilibria when $\delta_{1}=\delta_{2}=5 / 6$.

A striking feature of Example 3.6 is that the equilibrium proposals are typically not Pareto optimal, but only weakly so. The only exception are cases where $\delta_{1}=2 / 3$ or $\delta_{2}=$ 

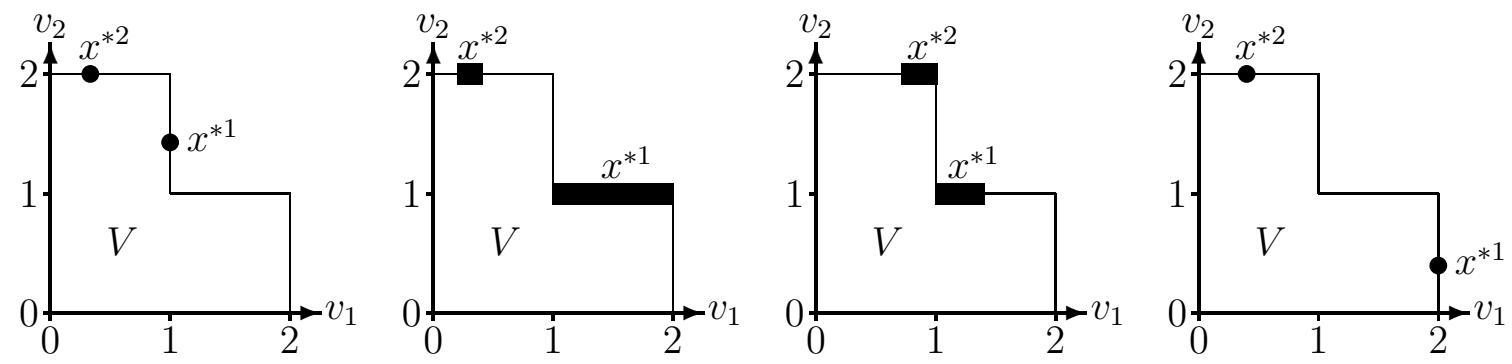

A: $\delta_{1}=\frac{1}{2}, \delta_{2}=\frac{5}{6}$

B: $\delta_{1}=\frac{1}{3}, \delta_{2}=\frac{2}{3}$

B: $\delta_{1}=\frac{5}{6}, \delta_{2}=\frac{2}{3}$

$\mathrm{C}: \delta_{1}=\frac{1}{3}, \delta_{2}=\frac{1}{3}$
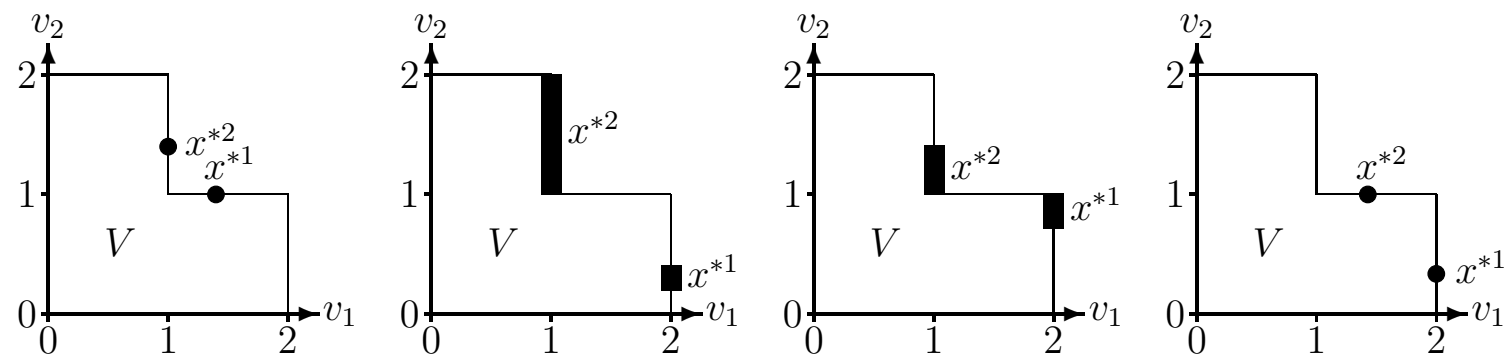

D: $\delta_{1}=\frac{5}{6}, \delta_{2}=\frac{5}{6}$

$\mathrm{E}: \delta_{1}=\frac{2}{3}, \delta_{2}=\frac{1}{3}$

$\mathrm{E}: \delta_{1}=\frac{2}{3}, \delta_{2}=\frac{5}{6}$

$\mathrm{F}: \delta_{1}=\frac{5}{6}, \delta_{2}=\frac{1}{2}$

Figure 3: Equilibrium proposals

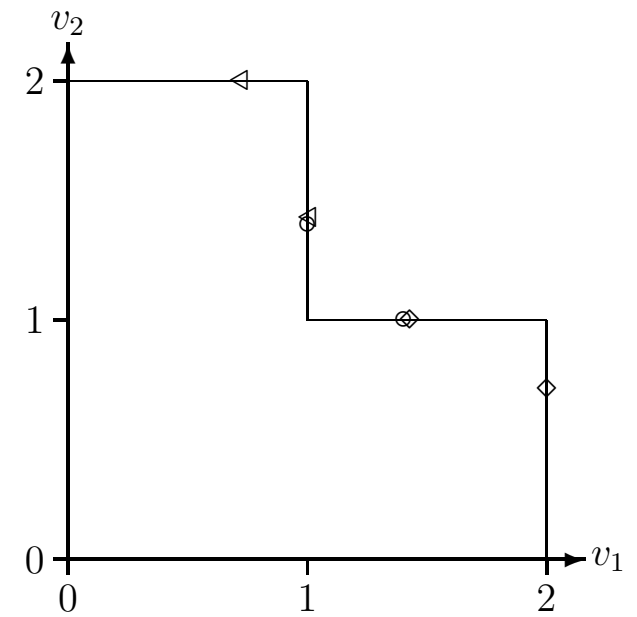

Figure 4: $\delta_{1}=\frac{5}{6}, \delta_{2}=\frac{5}{6}: \mathrm{A} \triangleleft, \mathrm{D} \circ, \mathrm{F} \diamond$ 
$2 / 3$, when there is a continuum of equilibria, and only one equilibrium in the continuum involves Pareto optimal proposals.

The most extreme equilibrium proposals for player 1 are $x^{* 1}=(2,0)$, which occurs when $\delta_{2}=0$ and $0 \leq \delta_{1}<2 / 3$, and $x^{* 1}=(1,2-\varepsilon)$ when $\delta_{2}=(4-2 \varepsilon) /(4-\varepsilon)$ and $0 \leq \delta_{1}<1$. Notice that in the latter equilibrium, player 1 may offer more to player 2 than to himself, even if player 1 is more patient than player 2 .

Another interesting feature of this example is that comparative statics may be counterintuitive. Consider the symmetric equilibrium corresponding to Region D when both players have discount rates exceeding $2 / 3$. In this region it holds that increasing patience worsens the bargaining position of a player. When discount rates converge to 1 , the equilibrium proposals of both players converge to $(1,1)$, a payoff that is weakly dominated for both players by alternative payoffs.

The example also demonstrates that equilibrium proposals are not even weakly Pareto optimal when compared to lotteries over feasible payoffs. When players become sufficiently patient, their equilibrium proposals become arbitrarily close to $(1,1)$. A lottery that selects the payoff $(2,1)$ with probability $1 / 2$ and the payoff $(1,2)$ with probability $1 / 2$ gives strictly higher utility to both players than both equilibrium proposals.

\section{A Characterization of All Stationary Subgame Per- fect Equilibria}

In this section we employ the additional assumption that each weakly Pareto-efficient point of $V_{+}$is Pareto efficient. Under this assumption we show that in any stationary subgame perfect equilibria of the game $\Gamma$ each player chooses a pure strategy on the equilibrium path, leading to a proposal which is immediately accepted by all the players. Furthermore, the vector of ex ante expected utilities in any stationary subgame perfect equilibrium of $\Gamma$ is a zero of the function $z$ as defined in Section 3.

Recall that a point $v \in V$ is said to be weakly Pareto-efficient if there is no point $x \in V$ such that $x_{i}>v_{i}$ for each $i \in N$. It is Pareto-efficient if there is no point $x \in V$ such that $x_{i} \geq v_{i}$ for each $i \in N$ with one strict inequality. We employ the following additional assumption:

(C) Each weakly Pareto-efficient point of $V_{+}$is Pareto efficient.

Assumption (C) is widely used in the literature and is often referred to as the condition of "non-levelness" of the relevant part of the boundary of the set $V$.

Let $\mathcal{B}$ be the set of Borel subsets of $V$. A stationary strategy for player $i$ can be summarized by a pair $\left(\mu^{i}, \tau^{i}\right)$ where $\mu^{i}: \mathcal{B} \rightarrow[0,1]$ is a probability measure and $\tau^{i}: V \rightarrow$ 
$[0,1]$ is a $\mathcal{B}$-measurable function. The number $\mu^{i}(B)$ is the probability for player $i$ to choose a proposal from the Borel set $B$, and $\tau^{i}(v)$ is the probability for player $i$ to accept a proposal $v$. Given a strategy profile $\left(\mu^{1}, \ldots, \mu^{n}, \tau^{1}, \ldots, \tau^{n}\right)$, let $\tau(v)=\tau^{1}(v) \times \cdots \times \tau^{n}(v)$ denote the probability that the point $v$ is unanimously accepted. Then the ex ante expected utility $u_{i}$ to player $i$ satisfies the following equation:

$$
u_{i}=\sum_{j=1}^{n} \theta_{j} \int_{V}\left[\tau(v) v_{i}+(1-\tau(v)) \delta_{i} u_{i}\right] d \mu^{j}(v) .
$$

THEOREM 4.1 Let the strategy profile $\sigma=\left(\mu^{1}, \ldots, \mu^{n}, \tau^{1}, \ldots, \tau^{n}\right)$ be an SSPE of $\Gamma$. Then for each $i \in N$ there exists a proposal $x^{i}$ in $V$ such that $\mu^{i}\left(\left\{x^{i}\right\}\right)=1$ and $\tau^{j}\left(x^{i}\right)=1$ for all $j \in N$. Furthermore, the ex ante expected utilities $u$ induced by $\sigma$ satisfy $z(u)=0$.

Proof: For $i \in N$, let $r_{i}=\delta_{i} u_{i}$. Define the sets $A$ and $B$ by

$$
A=\bigcap_{i \in N}\left\{v \in V \mid v_{i} \geq r_{i}\right\} \text { and } B=\bigcap_{i \in N}\left\{v \in V \mid v_{i}>r_{i}\right\} .
$$

STEP 0: $u_{i} \geq 0$ for each $i \in N$. Indeed, rejecting any proposal yields a player a utility of zero irrespective of the strategies of other players. Thus, in any Nash equilibrium of the game $\Gamma$ the utility to any player is at least zero.

SteP 1: If $v \in V$ is such that $\tau(v)>0$, then $v \in A$.

Suppose $\tau(v)>0$ and consider a history $h$ at time period 0 after which player $i$ has to respond to the proposal $v$. Notice that according to the strategy profile $\sigma$ all players accept $v$ with strictly positive probability. A rejection of $v$ by player $i$ yields him a utility of $r_{i}$, while accepting it yields a utility of $v_{i}$ with some positive probability and $r_{i}$ with the complementary probability. Since, according to the subgame perfect equilibrium strategy $\sigma$, player $i$ accepts $v$ with positive probability, we must have the inequality $v_{i} \geq r_{i}$.

Step 2: If $v \in B$, then $\tau(v)=1$.

Suppose without loss of generality that the players respond in the sequence $1, \ldots, n$. Consider a history $h$ at time period 0 after which player $n$ has to respond to the proposal $v$. All players preceding player $n$ have accepted the proposal $v$, because otherwise player $n$ is not requested to cast his vote by definition of the game $\Gamma$. Accepting $v$ by player $n$ yields him a utility of $v_{n}$, while rejecting it leads to a utility of $\delta_{n} u_{n}=r_{n}$. Thus player $n$ has to accept $v$ with probability 1 . We conclude that that $\tau_{n}(v)=1$.

Suppose that $\tau_{i+1}(v)=\cdots=\tau_{n}(v)=1$ for some $i$. Consider a history $h$ at time period 0 after which player $i$ has to respond to the proposal $v$. According to the definition of the game $\Gamma$, the players preceding $i$ in the response sequence have all accepted the proposal $v$. The players $i+1, \ldots, n$ will accept the proposal $v$ with probability 1 by the induction hypothesis, if player $i$ accepts it. Thus accepting $v$ by player $i$ yields a utility of $v_{i}$, while rejecting it gives a utility of $\delta_{i} u_{i}=r_{i}$. We conclude that $\tau_{i}(v)=1$. 
STEP 3: The set $B$ is non-empty.

Suppose $B$ is an empty set. We show next that then $A \subset\{r\}$. For suppose the set $A$ contains a point $v$ other than $r$. Then the point $r$ is not Pareto-efficient and, by Assumption (C), it is not weakly Pareto-efficient. It follows that there is a point $v \in V$ such that $v_{i}>r_{i}$ for each $i \in N$. But such a point $v$ is an element of the set $B$, a contradiction. This establishes that the set $A$ is either empty or it contains the point $r$ alone.

For each $i \in N$ and each $v \in V$, it holds that $\tau(v) v_{i}+(1-\tau(v)) \delta_{i} u_{i}=\delta_{i} u_{i}$, since it follows from Step 1 that if $\tau(v)>0$, then $v=r$, whereas the equality is trivial when $\tau(v)=0$. Using Equation 4.1 we find that $u_{i}=\delta_{i} u_{i}$ for each $i \in N$. We conclude that $u=0$. But 0 belongs to the interior of $V$ by Assumption (A), which implies that $B=\cap\left\{v \in V \mid v_{i}>0\right\}$ is a non-empty set, a contradiction.

Step 4: The set $A$ equals the closure of $B$.

Take $v \in A$ and an open neighborhood $O$ of $v$. We must show that the intersection $O \cap B$ is non-empty. Consider the point $y=(1-\lambda) v+\lambda r$ for some $\lambda \in(0,1)$ chosen small enough such that $y$ lies in the set $O$. Since $r_{i} \leq y_{i} \leq v_{i}$ for each $i \in N$ and since $V$ is comprehensive from below by Assumption (A), $y \in A$. The point $y$ is not Pareto-efficient. Indeed, if $y=v$, then $y=r$, which is not Pareto-efficient because the set $B$ is non-empty. And if $y$ is not equal to $v$, it is dominated by $v$. Hence, by Assumption (C), the point $y$ is not weakly Pareto-efficient. Thus there is a point $x \in V$ such that $y_{i}<x_{i}$ for all $i \in N$. Consider the point $x(\epsilon)=\epsilon x+(1-\epsilon) y$ for $0<\epsilon<1$. Since $r_{i} \leq y_{i}<x_{i}(\epsilon) \leq x_{i}$ for each $i \in N$ and since $V$ is comprehensive from below, $x(\epsilon) \in B$. And since $y \in O$, one can choose $\epsilon$ small enough so that $x(\epsilon) \in O$.

Step 5: The set $X^{i}=\arg \max _{v \in A} v_{i}$ contains a single element, say $x^{i}$. The point $x^{i}$ lies on the boundary of $V$ and $x_{j}^{i}=r_{j}$ for each $j \in N \backslash\{i\}$.

The set $X^{i}$ is non-empty, because $A$ is a compact set. Take any point $x \in X^{i}$ and suppose $r_{j}<x_{j}$ for some $j \in N \backslash\{i\}$. Define the point $v$ by the equation

$$
v_{k}= \begin{cases}x_{k}, & \text { if } k \in N \backslash\{j\}, \\ r_{j}, & \text { if } k=j .\end{cases}
$$

Thus $v_{k} \leq x_{k}$ with strict inequality for $k=j$. Since $V$ is comprehensive from below by Assumption (A), $v \in V$. Furthermore, the point $v$ is not Pareto-efficient being dominated by $x$. Hence by Assumption $(\mathrm{C})$ it is not weakly Pareto-efficient. Therefore, there exists a point $y \in V$ such that $v_{k}<y_{k}$ for each $k \in N$. Since $r_{k} \leq x_{k}=v_{k}<y_{k}$ for each $k \in N \backslash\{j\}$ and $r_{j}=v_{j}<y_{j}$, the point $y$ is an element of $A$. Furthermore $x_{i}<y_{i}$, contradicting the choice of $x$ in $X^{i}$. 
We have thus shown that, for each $x \in X^{i}$, for each $j \in N \backslash\{i\}, x_{j}=r_{j}$. It now follows at once that $X^{i}$ contains a single element.

Finally, if $x^{i}$ were not a boundary point of $V$, there would be a point $y \in V$ such that $x_{k}<y_{k}$ for all $k \in N$. Such a point $y$ would then be in the set $A$, contradicting the fact that $x^{i}$ is an element of $X^{i}$.

STEP 6: It holds that $\mu^{i}\left(\left\{x^{i}\right\}\right)=1$ and $\tau\left(x^{i}\right)=1$.

Consider a history $h$ at time period 0 after which player $i$ has to make a proposal and let $q_{i}$ denote the utility of player $i$ in the corresponding subgame of $\Gamma$. By Step 2, any point $v$ of $B$ is accepted with probability 1 . Hence, $q_{i} \geq v_{i}$ for each $v \in B$. Since by Step 4 the set $A$ is a closure of $B$, we must have $q_{i} \geq v_{i}$ for each $v \in A$, and therefore $q_{i} \geq x_{i}^{i}$.

For a natural number $m$, let $A_{m}=\left\{v \in A \mid v_{i} \geq x_{i}^{i}-1 / m\right\}$. Notice that $r_{i}<v_{i} \leq x_{i}^{i}$ for each $v \in B$, so in particular $r_{i}<x_{i}^{i}$. Take an $m$ large enough so that $r_{i}<x_{i}^{i}-1 / m$. Let $q_{i}(v)=\tau(v) v_{i}+(1-\tau(v)) r_{i}$ be the utility to player $i$ from proposing the point $v \in V$. If $v \in V \backslash A$, it is rejected with probability one by Step 1 , so $q_{i}(v)=r_{i}<x_{i}^{i}-1 / m$. If $v \in A \backslash A_{m}$, then $q_{i}(v)<x_{i}^{i}-1 / m$, because in this case both $v_{i}$ and $r_{i}$ are smaller than $x_{i}^{i}-1 / m$. And if $v \in A_{m}$, then $q_{i}(v) \leq x_{i}^{i}$, because $v_{i} \leq x_{i}^{i}$. Thus

$$
q_{i}=\int_{V} q_{i}(v) d \mu^{i}(v) \leq \mu\left(V \backslash A_{m}\right)\left(x_{i}^{i}-\frac{1}{m}\right)+\mu\left(A_{m}\right) x_{i}^{i} \leq x_{i}^{i}-\mu\left(V \backslash A_{m}\right) \frac{1}{m} .
$$

But since $x_{i}^{i} \leq q_{i}$, we conclude that $\mu\left(V \backslash A_{m}\right)=0$. It follows by continuity of a probability measure that $\mu\left(\left\{x^{i}\right\}\right)=\mu\left(\cap_{m \in \mathbb{N}} A_{m}\right)=\lim _{m \rightarrow \infty} \mu\left(A_{m}\right)=1$.

Finally, for $m$ large enough so that $r_{i}<x_{i}^{i}-1 / m$,

$$
\begin{aligned}
x_{i}^{i} \leq q_{i} & =\tau\left(x^{i}\right) x_{i}^{i}+\left(1-\tau\left(x^{i}\right)\right) r_{i} \\
& \leq \tau\left(x^{i}\right) x_{i}^{i}+\left(1-\tau\left(x^{i}\right)\right)\left(x_{i}^{i}-\frac{1}{m}\right)=x_{i}^{i}-\left(1-\tau\left(x^{i}\right)\right) \frac{1}{m},
\end{aligned}
$$

which shows that $\tau\left(x^{i}\right)=1$.

STEP 7: The vector of ex ante expected utilities $u$ satisfies $z(u)=0$.

By Step 5 we have $x_{j}^{i}=\delta_{j} u_{j}$ for each $j \neq i$. Equation 4.1 now reads

$$
u_{i}=\sum_{j=1}^{n} \theta_{j} x_{i}^{j}=\theta_{i} x_{i}^{i}+\left(1-\theta_{i}\right) \delta_{i} u_{i} .
$$

We thus obtain $x_{i}^{i}=\alpha_{i} u_{i}$, where $\alpha_{i}$ is as defined in Section 3. Therefore $x^{i}=p^{i}(u)$. And, also by Step 5 , the point $x^{i}$ lies in the boundary of the set $V$, so $z\left(p^{i}(u)\right)=0$, as desired.

Q.E.D. 


\section{Conclusion}

The presence of non-convexities poses many problems in economic modeling. Such problems are usually resolved by the use of lotteries, which convexify the problem. In this paper we argue that the canonical model of non-cooperative bargaining does not involve such difficulties. Even when the set of feasible payoffs is not convex, there exists a subgame perfect equilibrium in pure stationary strategies. At such an equilibrium, there is no delay. The only assumptions on the set of feasible payoffs that are needed for this result are that the set of feasible payoffs is closed, comprehensive from below, and that its restriction to the individually rational payoffs is bounded.

When we impose the mild additional requirement that the weak Pareto optimal payoffs in the set of feasible payoffs coincide with the Pareto optimal ones, we also obtain the reverse result that all subgame perfect equilibria in stationary strategies use pure strategies on the equilibrium path and lead to absence of delay. When players bargaining in a nonconvex environment, it is not only the case that equilibria without randomization exist, but even stronger, there are no equilibria where randomization is used. Nevertheless, it is easy to construct example where stationary mixed strategy profiles would lead to Pareto improvements of the equilibrium utilities.

Here we have restricted ourselves to the canonical model of non-cooperative bargaining. It is natural to examine to what extent our main results are also valid in extensions of this basic model, allowing for more general proposer selection and cake processes as studied for instance in Merlo and Wilson (1995). We have studied the classical case with unanimous acceptance of proposals. A generalization of our results to the case with a general set of decisive coalitions as in Banks and Duggan (2000) will fail to hold, since in such a setting pure strategy equilibria may fail to exist even when sets of feasible payoffs are convex. The assumption of unanimous approval seems therefore to be crucial for the existence of pure strategy equilibria and the absence of randomization in general environments.

\section{References}

Banks, J., And J. Duggan (2000), "A Bargaining Model of Collective Choice," American Political Science Review, 94, 73-88.

Conley, J.P., And S. Wilkie (1995), "Implementing the Nash Extension Theorem to Nonconvex Bargaining Problems," Economic Design, 1, 205-216.

Conley, J.P., And S. Wilkie (1996), "An Extension of the Nash Bargaining Solution to Nonconvex Problems," Games and Economic Behavior, 13, 26-38. 
Fudenberg, D., And J. Tirole (1991), Game Theory, MIT Press, Cambridge, Massachusetts.

Herrero, M.J. (1989), "The Nash Program: Non-convex Bargaining Problems," Journal of Economic Theory, 49, 266-277.

Kaneko, M. (1980), "An Extension of the Nash Bargaining Problem and the Nash Social Welfare Function," Theory and Decision, 12, 135-148.

Kahneman, D., And A. Tversky (1979), "Prospect Theory: An Analysis of Decisions Under Risk," Econometrica, 47, 263-291.

Kalandrakis, T. (2004), "Equilibria in Sequential Bargaining Games as Solutions to Systems of Equations," Economics Letters, 84, 407-411.

Kalandrakis, T. (2006), "Regularity of Pure Strategy Equilibrium Points in a Class of Bargaining Games," Economic Theory, 28, 309-329.

Mariotti, M. (1997), "Extending Nash's Axioms to Non-convex Problems," Games and Economic Behavior, 22, 377-383.

Merlo, A., And C. Wilson (1995), "A Stochastic Model of Sequential Bargaining with Complete Information," Econometrica, 63, 371-399.

Rubinstein, A. (1982), "Perfect Equilibrium in a Bargaining Model," Econometrica, 50, $97-109$.

ScARF, H.E. (1994), "The Allocation of Resources in the Presence of Indivisibilities," Journal of Economic Perspectives, 8, 111-128.

Xu, Y., And N. Yoshinara (2006), "Alternative Characterizations of Three Bargaining Solutions for Nonconvex Problems," Games and Economic Behavior, 57, 86-92.

Zhou, L. (1997), "The Nash Bargaining Theory with Non-convex Problems," Econometrica, 65, 681-686. 